\title{
Accuracy Evaluation of Classical Integer Order and Direct Non-integer Order Based Numerical Algorithms of Non-integer Order Derivatives and Integrals Computations
}

\author{
Dariusz W. Brzeziński \\ Institute of Applied Computer Science \\ Lodz University of Technology \\ 18/22 Stefanowskiego St., 90-924 Łódź, Poland \\ Email:dbrzezinski@kis.p.lodz.pl
}

\author{
Piotr Ostalczyk \\ Institute of Applied Computer Science \\ Lodz University of Technology \\ 18/22 Stefanowskiego St., 90-924 Łódź, Poland \\ Email:piotr.ostalczyk@p.lodz.pl
}

\begin{abstract}
In this paper the authors evaluate in context of numerical calculations accuracy classical integer order and direct non-integer based order numerical algorithms of non-integer orders derivatives and integrals computations. Classical integer order based algorithm involves integer and fractional order differentiation and integration operators concatenation to obtain non-integer order. Riemann-Liouville and Caputo formulas are applied to obtain directly derivatives and integrals of non-integer orders. The following accuracy comparison analysis enables to answer the question, which algorithm of the two is burdened with lower computational error. The accuracy is estimated applying non-integer order derivatives and integrals computational formulas of some elementary functions available in the literature of the subject.
\end{abstract}

\section{INTRODUCTION}

T HERE are many formulas which can be applied to compute directly derivatives and integrals of non-integer orders [8], [9], [10], [11], [12], [14]. They include RiemannLiouville non-integer (fractional) order integral/derivative and Caputo non-integer (fractional) derivative. However, noninteger order derivatives and integrals can be also computed applying integer and fractional order differentiation and integration operators concatenation. Classical integer order derivatives and integrals can be obtained applying well known numerical techniques. To calculate fractional order derivatives and integrals, Riemann-Liouville/Caputo formulas can be applied. The question, the authors want to answer is, which of the algorithms enables to obtain more accurate values of example non-integer derivatives and integrals of some elementary functions.

The paper is divided into the following parts: at the beginning the authors present details of the applied operators of non-integer (fractional) order differentiation and integration, followed by detailed description of the integer and fractional order differentiation and integration operators concatenation and the explanation of their practical numerical implementations. The final part of the paper include accuracy comparison analysis of example non-integer order derivatives and integrals of some elementary functions. The values assumed as exact for the accuracy comparison are calculated applying formulas of non-integer order derivatives and integrals available in the literature of the subject [11], [14].

\section{Mathematical Preliminaries}

Non-integer (fractional) order integration and differentiation operators include:

- Riemann-Liouville Fractional Order Integral

$$
{ }_{t_{0}}^{R L} \mathbf{I}_{t}^{(\nu)}=\frac{1}{\Gamma(\nu)} \int_{t_{0}}^{t} \frac{f(\tau)}{(t-\tau)^{1-\nu}} d \tau
$$

- Riemann-Liouville Fractional Order Derivative

$$
{ }_{t_{0}}^{R L} \mathrm{D}_{t}^{(\nu)} f(t)=\frac{1}{\Gamma(n-\nu)}\left(\frac{d}{d t}\right)^{n} \int_{t_{0}}^{t} \frac{f(\tau)}{(t-\tau)^{1-\nu}} d \tau
$$

- Caputo Fractional Derivative

$$
{ }_{0}^{C} \mathrm{D}_{t}^{(\nu)} f(t)=\frac{1}{\Gamma(n-\nu)} \int_{0}^{t} \frac{f^{(n)}(\tau)}{(t-\tau)^{1-\nu}} d \tau
$$

Formulas (2) and (3) are related by

$$
{ }_{t_{0}}^{R L} D_{t}^{(\nu)} f(t)={ }_{0}^{C} D_{t}^{(\nu)} f(t)+\sum_{k=0}^{n-1} \frac{t^{k-\nu}}{\Gamma(k-\nu+1)} f^{(k)}(0)
$$

where: $n-1<\nu<n \in N=\{1,2, \ldots\}, \nu \in R$ is the order of fractional integral/derivative, $f^{(n)}(\tau)=\frac{d^{n} f(\tau)}{d \tau^{n}}$ is the classical derivative of integer order, $\Gamma(x)=\int_{0}^{\infty} e^{-t} t^{x-1} d t$ is the gamma function and $t$ satisfies the following conditions $-\infty<t_{0}<t<\infty,\lfloor n\rfloor=n+\nu$. 


\section{Applied Methods of Integration AND DIFFERENTIATION}

A. Applied Methods for Classical Integer Orders Derivatives and Integrals Computations

- Classical integer order derivatives are calculated applying well known numerical methods involving 5 point stencil Central Diffidences.

- Classical integrals of the integer orders are obtained applying well known and efficient methods of numerical integration:

\section{- Gauss-Kronrod Quadrature (denoted as GKr)}

* The method is a very efficient modification of well known Gauss-Legendre Rule. It uses so called the Gauss-Kronrod Pairs. For example the G30/K61 pair includes the nodes of the 30-point GaussLegendre Quadrature + 31 new ones and all 61 different coefficients.

* The advantages of the method is its efficiency and high accuracy obtained with only some dozens of sampling points if applied to precisely selected type of integrand. The main disadvantages are: a complex method of nodes and weight calculations, high precision input data requirement and indept knowledge about the method application to actually obtain high accuracy results.

- Midpoint Rule (denoted as NCm)

* The method is a very efficient modification of the rectangular rule. In the midpoint rule the sample point is taken from the middle of each subinterval. This feature enable the application of the method to the integrands with singularities at the end point of the integration range.

* The method can be applied to any integrand, because the weight function equals 1 and the nodes of quadrature are of equal width. The accuracy of calculations should theoretically increase while increasing the amount of subintervals, for which the integration range is divided into. However mechanical increase of the amount of subintervals often leads to small accuracy increase and big increase in computational complexity.

All further details regarding integer order derivatives and integrals methods of computations can be found in available literature of the subject [1], [2], [3], [4], [5]. There is only presented, on Figs 2-3 indicative accuracy of the applied methods.

B. Applied New Efficient High-accuracy Methods of Integration for Non-integer and Fractional Orders Derivatives and Integrals Computations

The integrands in formulas (1)-(3) are difficult to integrate due to singularity at the end of integration range. The difficulty rises as the order of calculated fractional order integral nears 0 and the order of fractional order derivative nears 1 . This feature makes inefficient widely known methods of numerical integration in context of accuracy of calculations. Additionally some of the fractional orders derivatives and integrals, mentioned in last sentence are not possible to compute with satisfactory accuracy at all (relative error exceeding 90-100 $\%)$.

Generally the problems associated with singularities in numerical integration are the most difficult to solve. In such cases high accuracy results can only be obtained by application of dedicated methods, as for example weighted type quadratures. This type of quadratures, however can only be applied to precisely selected types of functions in unmodified form. It is forced by their association with the corresponding weights. To obtain high accuracy results, either the integrand must satisfy conditions of a particular quadrature application requirements or the quadrature must be adopted to a particular integrand. Choosing the second method, the authors of the paper developed precise modifications to the existing numerical methods of integration: Gauss-Jacobi Quadrature and Double Exponential Quadrature, which initially were developed for integer order integration only.

Modified version of Gauss-Jacobi Quadrature have adopted weight function for fractional order derivatives and integrals computations (the method is denoted in the paper as GJ). Double Exponential Quadrature (denoted as DE) [16], [17] on the other hand involves hyperbolic functions substitution in independent variable transformation in integrand and trapezoidal rule applied to the transformed integrand. Application of the methods enables to obtain high accuracy computations results of fractional order derivatives and integrals [6], [7].

Important remarks:

- In the case of the non-integer (fractional) order differentiation and integration operators (1)-(3), regardless if there is integral or derivative to calculate, we always apply the integration operator.

- The non-integer (fractional) order differentiation operators (1)-(3) unlike integer order differentiation operators, are non-local operators. They are not calculated applying the values of the neighbor function points, but from the whole range of differentiation. This can be beneficial in case of a physical process analysis, because we take into consideration its history from the beginning, however this feature increases significantly the complexity of numerical calculations. Higher complexity of calculations influences negatively the accuracy of input data for each part of calculations. Inaccuracies in input data are the classical cause for numerical calculations accuracy decrease.

C. Concatenation of Operators of Integer and Fractional Order Differentiation and Integration as a Obtain Method of Non-integer Order

Scientifically interesting calculation method of non-integer order derivatives and integrals computations is application of concatenation of integer and fractional order differentiation and integration operators.

The practical operations of concatenations are related with fractional and integer order operators. Some of them are trivial, 


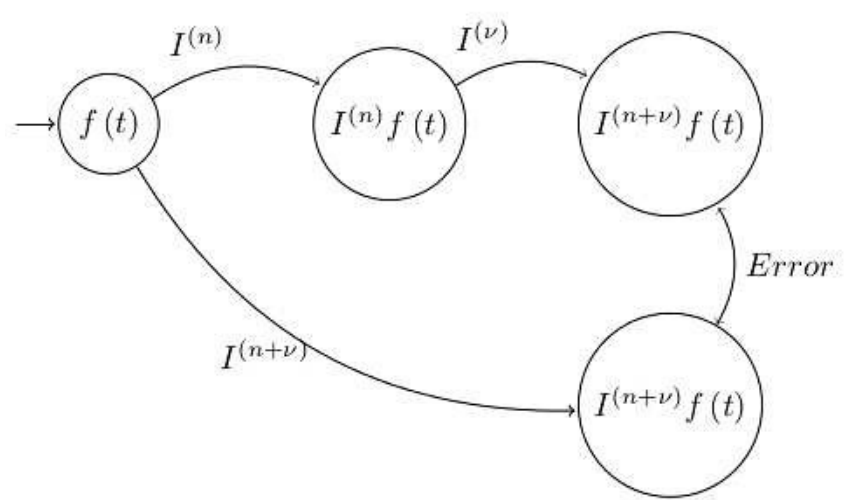

Fig. 1. Accuracy Estimation Based on Integer and Fractional Orders Integration Operators Concatenation

some require additional assumptions. There is always assumed the existence of the corresponding derivatives and integrals and that the operations of concatenation are always performed on the same definitive integration range.

Interesting concatenations combination of integer and fractional orders differentiation and integration in context of the paper's subject include:

- Fractional order derivative of integer order derivative

$$
\mathrm{D}^{(\nu)}\left(\mathrm{D}^{(n)} f(t)\right)=\mathrm{D}^{(n+\nu)} f(t)
$$

- Fractional order integral of integer order integral

$$
\mathbf{I}^{(\nu)}\left(\mathbf{I}^{(n)} f(t)\right)=\mathbf{I}^{(n+\nu)} f(t)
$$

where $n$ is classical integer order derivative or integral, $\nu \geq 0$ and $t \geq 0$ (see Figure 1 for visualization).

Comprehensive mathematical calculations to the subject can be found in [14].

In the works of [9] and [10] discussed properties (5) and (6) are named Law of Exponents, because they relate to the operations performed on the exponents of the fractional and integer order operators (1)-(3). The Law of Exponents is generally true for the fractional integration operators. It is also valid for fractional order differentiation operators [14]. Still it is well known [10] and [9] in $\S I V .6$ Law of Exponents, that there exist functions for which fractional order differentiation operator (3) do not satisfy (5). However, the authors of the following paper focus only on functions, for which concatenations (5)-(6) are possible, in this case $f\left(t_{0}\right)=0$.

\section{Evaluation Details}

The description of a general method of the fractional order differentiation and integration operators concatenation is presented in [10], [9]. It is expressed by the following formula

$$
\begin{aligned}
I^{(\nu)}\left[I^{(n)} f(t)\right]=\frac{1}{\Gamma(\nu) \cdot \Gamma(n)} \int_{0}^{t}(t-x)^{\nu-1} \cdot \\
\cdot\left[\int_{0}^{x} f(y) d y\right] d x
\end{aligned}
$$

We substitute $x=k * h$ in inner integral, where $k$ is the amount of subintervals, which the integration range is divided into, and $h$ is the width of each interval. As a result we obtain the values of the inner integral in $h$-spaced intervals. This values serve as input function $f(x)$ for the outside integral.

The integration methods applied in the following research require precisely sampled function's values in the nodes points and their synchronization with the corresponding weights. Sampling the function in equally spaced points and then interpolating the values of the function in the nodes points does not comply with its function sufficiently. There must be applied a different approach, which is presented as Algorithm 1.

Algorithm 1 Concatenation of integer and fractional order differentiation and integration operators. Nodes of a quadrature for $I^{(\nu)}$ as an integration step of $I^{(n)}$.

Step 1. Select fractional orders to concatenate $I^{(n)}$ and $I^{(\nu)}$. The $I^{(n)}$ is the inner integral and the $I^{(\nu)}$ is the outer one.

Step 2. Calculate quadrature nodes and weights for the $I^{(\nu)}$.

Step 3. Calculate $I^{(n)}$ applying as step the points of the nodes from Step 2.

Step 4. Calculate $I^{(\nu)}$ applying as inputting as $f(x)$ the values obtained in Step 3.

1) Arbitrary Precision in Numerical Calculations: To overcome the bottlenecks of the double precision computer arithmetic and to increase overall computations accuracy [18], two arbitrary precision programming libraries together with a $\mathrm{C}++$ wrapper [22] are applied:

MPFR [21] is an arbitrary precision package for $\mathrm{C}$ language and is based on GMP [20].

MPFR supports arbitrary precision floating point variables. It also provides an exact rounding of all implemented operations and mathematical functions [19].

\section{A. Testing Functions}

The integrand in formulas (1)-(3) consists of two factors: the first factor, so called core and the second one, which is the actual function, of which there is non-integer (fractional) order derivative or integral to calculate.

The core has the biggest influence on the shape of the integrand and on the difficulty level of integration. In this respect, the actual function to integrate contributes only to a minimal extent.

Due to this, the authors decide it is enough to select two functions for testing purposes:

- Power function

$$
f(t)=\left(t-t_{0}\right)^{p}, t_{0}=0, p=0.5, t \in(0,1)
$$

- Exponential function

$$
f(t)=e^{a t} \mathbf{I}(t), a=0.5, t \in(0,1)
$$




\section{B. Accuracy Estimation of Computations}

Accuracy estimation is performed on the basis of the noninteger (fractional) order derivatives and integrals formulas (10)-(13) available in the literature of the subject [11], [14]. Due to the fact, that they are in fact computational formulas, there must be taken into consideration some calculations error, although very small.

Assuming $D^{(-\nu)}=I^{(\nu)}$.

- Power Function (8):

- Fractional integral

$$
{ }_{t_{0}} \mathrm{D}_{t}^{(-\nu)} f(t)=\frac{\Gamma(p+1)}{\Gamma(p+\nu+1)}\left(t-t_{0}\right)^{p+v} .
$$

- Fractional derivative

$$
{ }_{t_{0}} \mathrm{D}_{t}^{(\nu)} f(t)=\frac{\Gamma(p+1)}{\Gamma(p+1-\nu)}\left(t-t_{0}\right)^{p-v} .
$$

- Exponential Function (9):

- Fractional integral

$$
{ }_{t_{0}} \mathrm{D}_{t}^{(-\nu)} f(t)=t^{\nu} \sum_{k=0}^{N} \frac{(a t)^{k}}{\Gamma(k+\nu+1)} .
$$

- Fractional derivative

$$
{ }_{t_{0}} \mathrm{D}_{t}^{(\nu)} f(t)=t^{-\nu} \sum_{k=0}^{N} \frac{(a t)^{k}}{\Gamma(k+1-\nu)} .
$$

\section{Accuracy Definition}

In the whole paper the accuracy is expressed as relative error in $\%$ in context of integration range

$$
e_{r}^{(t)}=\left(1-\frac{v_{c}}{v_{e}}\right) \cdot 100 \%
$$

where $v_{c}$ denotes calculated value, $v_{e}$ a value assumed as exact one and $t_{0}, t$ is integration range.

\section{COMPARISON ANALYSiS}

\section{A. General remarks}

The methods of numerical integration developed by the authors of the paper, to their best knowledge, are the only numerical methods of integration available at the moment, applying which it is possible to obtain high accuracy results calculating non-integer (fractional) derivatives and integrals:

- GJ method delivers high accuracy results with average accuracy above $10^{-80}$ mark. The order of the derivative and integral and integration range has no impact on accuracy. Actually, the method increases offered accuracy in cases, in which traditionally methods decrease it. The method requires 4-64 sampling points to reach average accuracy abilities. The type of integrated function influences only slightly the final results.

- DE is able to deliver $10^{-50}$ average accuracy level for fractional integrals of orders greater than 0.5 and fractional derivatives smaller that 0.5 with $600-1000$ sampling points. The methods is in general more dependable on range and type of the integrated function.

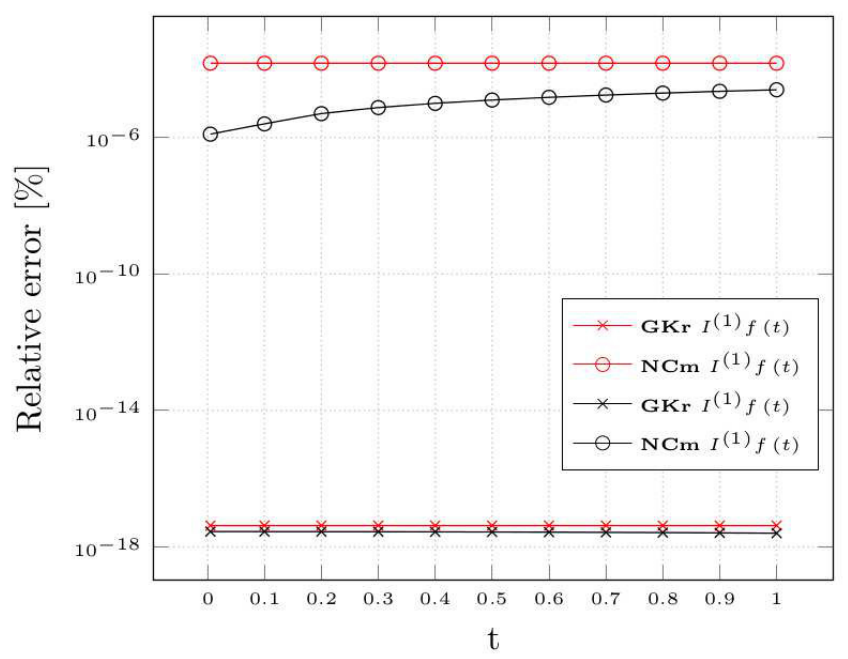

Fig. 2. Integer Order Integration results for function (8) in red and function (9) in black

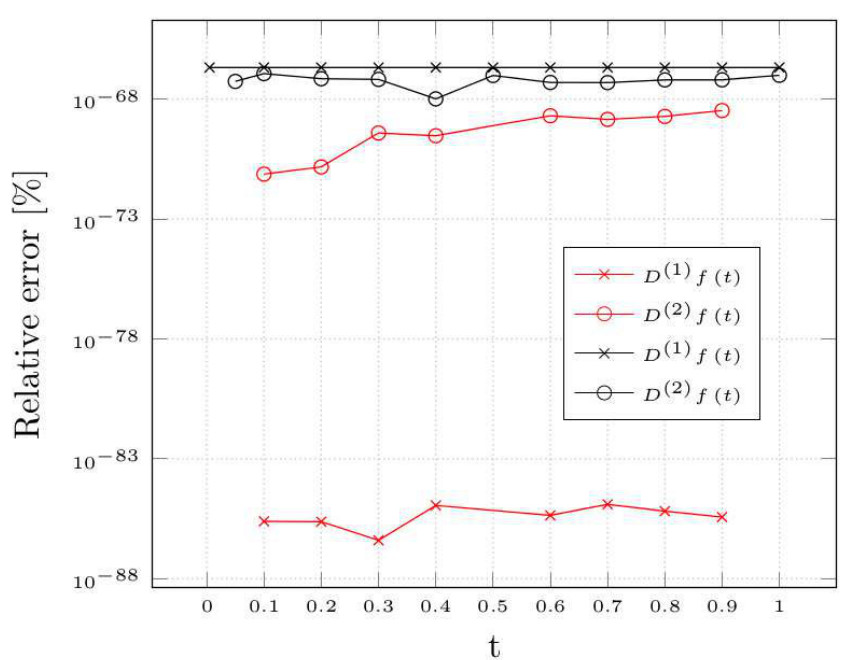

Fig. 3. Integer Order Differentiation results for function (8) in red and function (9) in black

\section{B. Accuracy of integer order integration and differentiation}

As it is presented on Fig. 2, GKr ensures enormous advantage over $\mathrm{NCm}$ in context of accuracy of integration. $\mathrm{GKr}$ achieves triple greater accuracy with only 61 sampling points. $\mathrm{NCm}$ was tested with 10000 sampling points. Further sampling points increase in case of $\mathrm{GKr}$ did not enable to obtain better results; actually, the accuracy deteriorated. In case of $\mathrm{NCm}$, further accuracy increase was very slow with each 10000 sampling points added, that it did not justified the further experimentation.

Application of $\mathrm{GKr}$ is the optimal choice for integration operators concatenation.

Application of 5-point Central Differences to obtain $1^{\text {st }}$ and $2^{\text {nd }}$ derivative resulted with high accuracy, almost error free results (See Fig. 3). Further points increase did not bring any 
accuracy increase.

Application of 5-point Central Differences is the optimal choice for differentiation operators concatenation.

\section{Accuracy of non-integer order integration and differentia- tion}

As it is presented on Figs 4-7 application of both developed methods of numerical integration GJ and DE to compute directly non-integer order derivatives and integrals enables to obtain highest possible results.

The accuracy of the integer and non-integer orders integration operators concatenation is limited to the accuracy possible to obtain applying integer order numerical integration methods, because it determines the accuracy of the input data for fractional order integration. For differentiation operators concatenation, the deteriorating amount of information about the integrated function during the operators concatenation affects the final accuracy. The loss of information understood in the sense of the input data accuracy decrease: during integer operator application the function is known in the entire range, i.e. it is available in the continuous form; during the fractional operator application the input function is available only in some earlier pre-calculated points, i.e. it is available in the discrete form only.

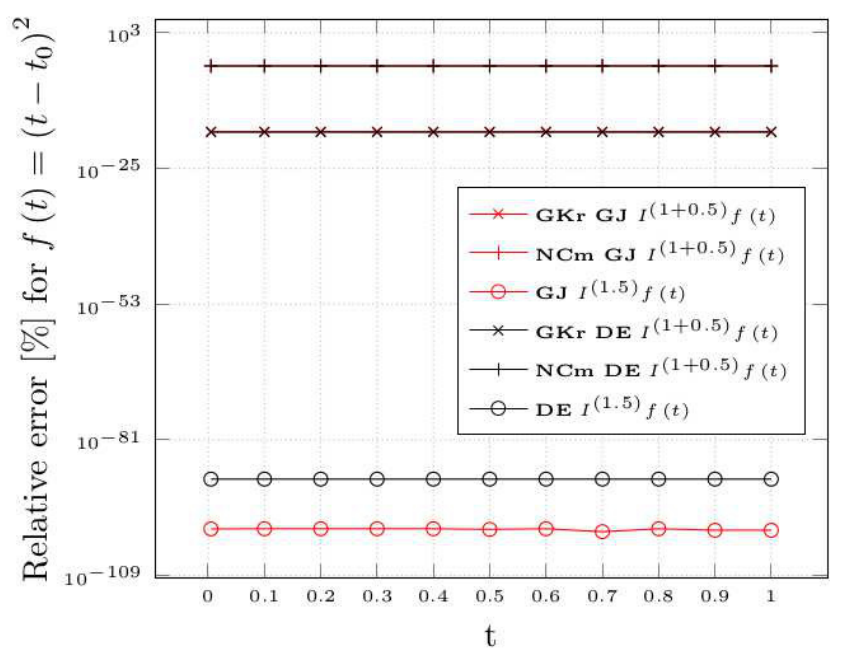

Fig. 4. Non-integer Order Integration Results for function (8). GKr and NCm applied to compute integer orders, GJ and DE to compute fractional and noninteger order

\section{CONCLUSION}

The purpose of the following research was to evaluate in context of computations accuracy two algorithms of noninteger order derivatives and integrals computations: direct numerical calculation of non-integer order derivatives and integrals and the non-integer orders of derivatives and integrals obtained by the application of concatenation of the integer and fractional orders operators of integration and differentiation.

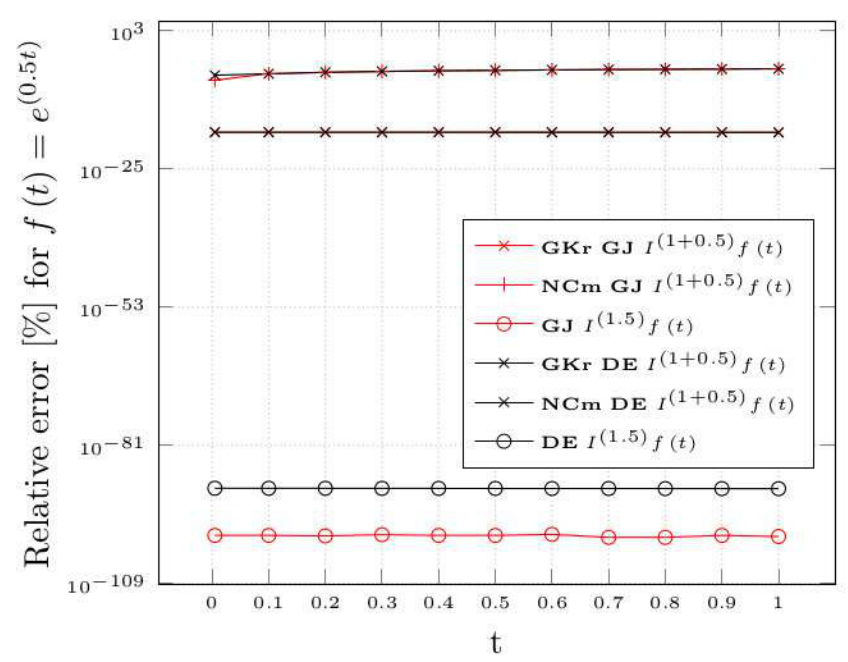

Fig. 5. Non-integer Order Integration Results for function (9) $\mathrm{GKr}$ and $\mathrm{NCm}$ applied to compute integer orders, GJ and DE to compute fractional and noninteger order

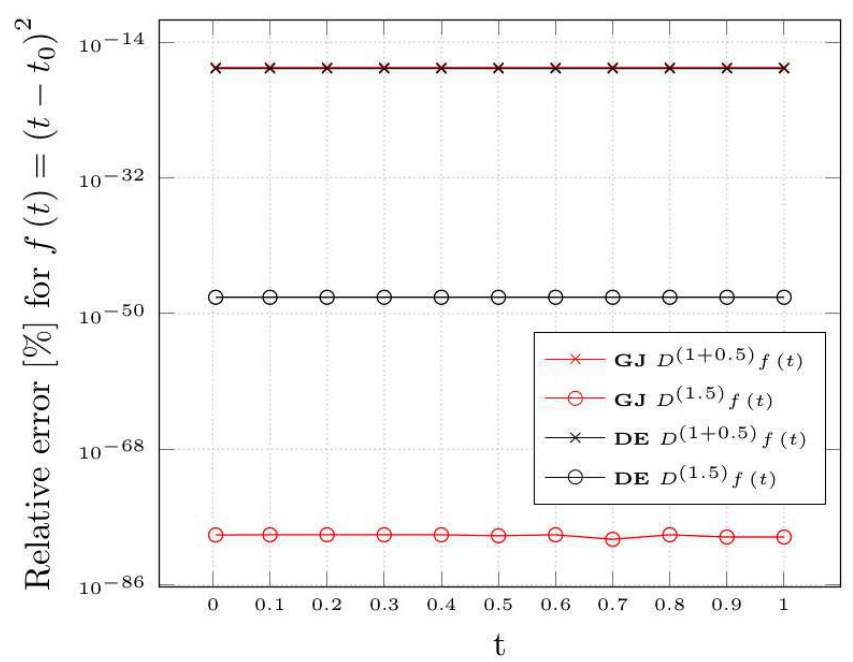

Fig. 6. Non-integer Order Differentiation Results for function (8)

There is no doubt, that direct calculation of non-integer orders derivatives and integrals applying both numerical methods, GJ and DE, developed by the authors of the paper for non-integer and fractional order derivatives and integrals computations applying formulas (1)-(3) are the methods to favorite if one want to obtain high accuracy results. The methods are efficient and their accuracy does not depend on order of the calculated derivative and integral or the integration range.

\section{REFERENCES}

[1] R.L. Burden, J.D. Faires, "Numerical Analysis“, 5 th. Ed., Brooks/Cole Cengage Learning, Boston, 2003.

[2] R.A. Krommer, Ch.W. Ueberhuber, "Computational Integration", SIAM, Philadelphia, 1986. 


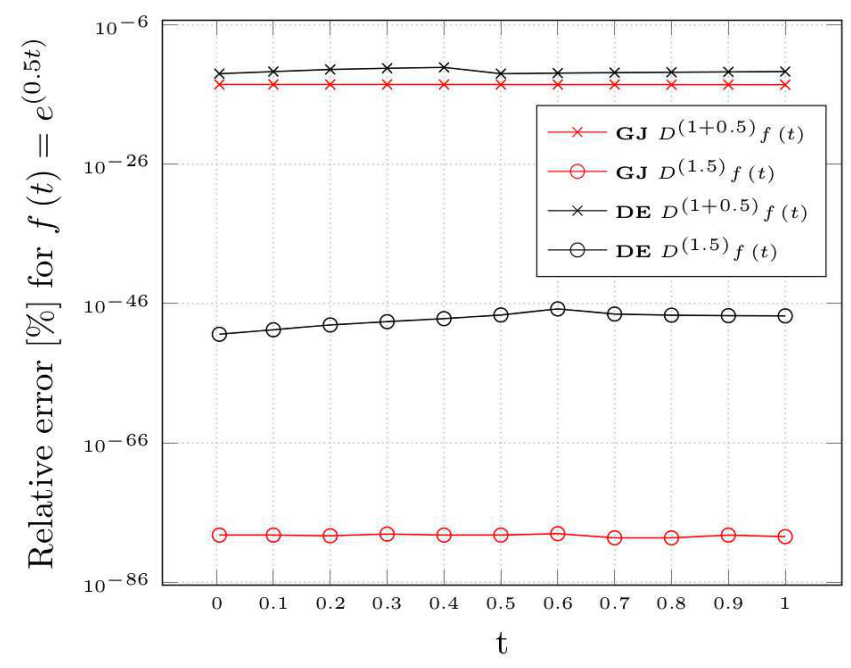

Fig. 7. Non-integer Order Differentiation Results for function (9)

[3] A.H. Stroud,D. Secrest, "Gaussian Quadrature Formulas", Prentice-Hall, Englewood Cliffs, NJ., 1966.

[4] P.K.Kythe, M.R.Schäferkotter, "Handbook of Computational Methods For Integration“, Chapman \& Hall/CRC, 2005.

[5] M. Hjort-Jensen, "Computational Physics", University of Oslo, 2009.

[6] D.W. Brzeziński and P. Ostalczyk, "High-accuracy Numerical Integration Methods for Fractional Order Derivatives and Integrals Computations", After reviews, awaits publication in Bulletin of the Polish Academy of Sciences Technical Sciences, vol. 4, 2014.

[7] D.W. Brzeziński and P. Ostalczyk, "Evaluation of Efficient Methods of Fractional Order Derivatives and Integrals Numerical Calculations", in Proceedings of the XIV Conference on System Modelling and Control, September 23-24 2013, Lódz, Poland, 2013.

[8] D. Baleanu, K. Diethelm, E. Scalas, and J.J. Trujillo, "Fractional Calculus Models and Numerical Methods,“ World Scientific Publishing Co.Pte. Ltd., Singapore, 2012.

[9] K.S. Miller and B. Ross, "An Introduction To The Fractional Calculus and Fractional Differential Equations", John Willey and Sons INC., New York,NY, 1993

[10] R. Gorenflo and F. Mainardi, "Fractional Calculus: Integral and Differential Equations of Fractional Order", in Fractals and Fractional Calculus in Continuum Mechanics, Springer-Verlag, Wien and New York, 1997.

[11] I. Podlubny, Fractional Differential Equations, Academic Press, San Diego, CA, 1999.

[12] K.B. Oldham, J. Spanier, "The Fractional Calculus. Theory and Applications of Differentiation and Integration to Arbitrary Order", Academic Press, 1974.

[13] A.A. Kilbas, H.M. Srivastava, J.J. Trujillo, "Theory and Applications of Fractional Differential Equations“, North Holland Mathematics Studies 204, Elsevier, 2006.

[14] P. Ostalczyk, Zarys rachunku różniczkowego i całkowego ułamkowych rzȩdów, Komitet Automatyki i Robotyki Polskiej Akademii Nauk, Wydawnictwo Politechniki Łódzkiej, Łódź, Poland, 2008.

[15] C. Schwartz, "Numerical Integration of Analytic Functions", in Journal of Computational Physics, vol. 4, pp. 19-29, 1969.

[16] H. Takahasi, Quadrature Formulas Obtained by Variable Transformation, Numerische Mathematik, nr 21, 1973.

[17] M. Mori, "Discovery of The Double Exponential Transformation and Its Developments“, publ. RIMS, Kyoto Univ., 41, pp. 897-935, 2005.

[18] J.M. Muller, N. Brisebarre, F. De Dinechin, C.P. Jeannerod, V. Lefevre, G. Melquiond, N.Revol, D. Stehle, D. and S. Torres, "Handbook of Floating-Point Arithmetic", Birkhauser Boston, New York,NY, 2010.

[19] K.R. Ghazi, V.Lefevre, P.Theveny and P.Zimmermann, "Why and how to use arbitrary precision" IEEE Computer Society, vol.12, nr 3, 2010, DOI Bookmark: http://doi.ieeecomputersociety.org/10.1109/MCSE.2010.73.

[20] The GNU Multiple Precision Arithmetic Library, https://gmplib.org/.
[21] The GNU Multiple Precision Floating-Point Reliable Library, https:// mpfr.org/.

[22] C++ wrapper for the GNU Multiple Precision Floating-Point Reliable Library, http://www.holoborodko.com/pavel/mpfr/.

[23] J. Waldvogel, "Towards A General Error Theory of the Trapezoidal Rule“, in Approximation and Computation, pp 267-282, Springer Verlag, W.Gautschi, G.Mastroianni and Th.M.Rassias (Eds.), 2011.

\section{APPENDIX}

Below there are presented the main ideas behind two new numerical methods of integration developed by the authors of the paper for non-integer (fractional) order derivatives and integrals computations mentioned in the paper.

\section{A. Double Exponential Formula}

The Double Exponential (DE) formula joins two applied techniques: the double exponential transformation applied to the initial integrand and the trapezoidal rule applied to the transformed integrand.

General idea standing behind the DE transformation which was proposed by Schwartz [15] and become known as the Tanh rule (since $x=\tanh (t)$ ) is as follows:

Let us consider the integral

$$
I=\int_{a}^{b} f(x) d x
$$

where $f(x)$ is integrable on interval $(a, b)$. The function $f(x)$ may have singularity $x=a, x=b$ or at both.

First we apply the following variable transformation

$$
x=\phi(t), \phi(-\infty)=a, \phi(\infty)=b .
$$

We obtain

$$
I=\int_{-\infty}^{\infty} f(\phi(t)) \phi^{\prime}(t) d t
$$

It is important that $\phi(t)$ possess the property such as $\phi^{\prime}(t)$ decreases its values to 0 at at least double exponential as $t \rightarrow$ $\pm \infty$, i.e.

$$
\left|\phi^{\prime}(t)\right| \rightarrow \exp (-c \exp (|t|))
$$

where $c$ is some constant.

After that, it is best to apply the trapezoidal formula with an equal mesh size to the transformed integrand expression [23], i.e.

$$
I=h \sum_{n=-\infty}^{\infty} f(\phi(n h)) \phi^{\prime}(n h)
$$

where $n h$ is sampling step.

Due to the property (15) truncation of the summation process can be done at some arbitrary chosen $n=-N_{-}$and $n=+N_{+}$, i.e.

$$
I=h \sum_{n=-N_{-}}^{N_{+}} f(\phi(n h)) \phi^{\prime}(n h),
$$

$N=N_{-}+N_{+}+1$, where $N$ states the amount of sampling points of the function.

Since $\phi^{\prime}(n h)$ as well as the whole expression $f(\phi(n h)) \phi^{\prime}(n h)$ converges to 0 at exponential rate at 
large $|n|$, the quadrature formula (16) is called the Double Exponential [16], [17].

Due to truncation of the summation process (16) at some arbitrary chosen $n=N_{-}, n=N_{+}$, function $f(x)$ can have singularities at $x=a$ and/or $x=b$ as long as it is integrable over the integration range.

There should be taken two kinds of errors into consideration when implementing the DE formula: discretization error, because we use the trapezoidal rule to approximate an integral and truncation error, because we truncate infinite sum at some $N$.The optimal strategy is to make both errors equal [17].

The subinterval width $h$, which defines the evaluation step and the number of sample points are key values in such strategy. The source [17] suggest the following value of $h$ for DE formula

$$
h \sim \frac{\log (2 \pi N \omega / c)}{N},
$$

where $c$ is some constant to be taken, usually 1 or $\pi / 2$ and $\omega$ is the distance to the nearest singularity of the integrand.

Correct selection of a function (17)-(19) with optimal properties enables to control the level of convergence of the whole transformed expression (16). The rate of convergence has enormous impact on accuracy, i.e. to rapid convergence decreases the accuracy [16].

The authors test three different transformations and selected (18) because of it optimal convergence rate for the purpose of the research, which is also suggested by the literature of the subject [17], [16].

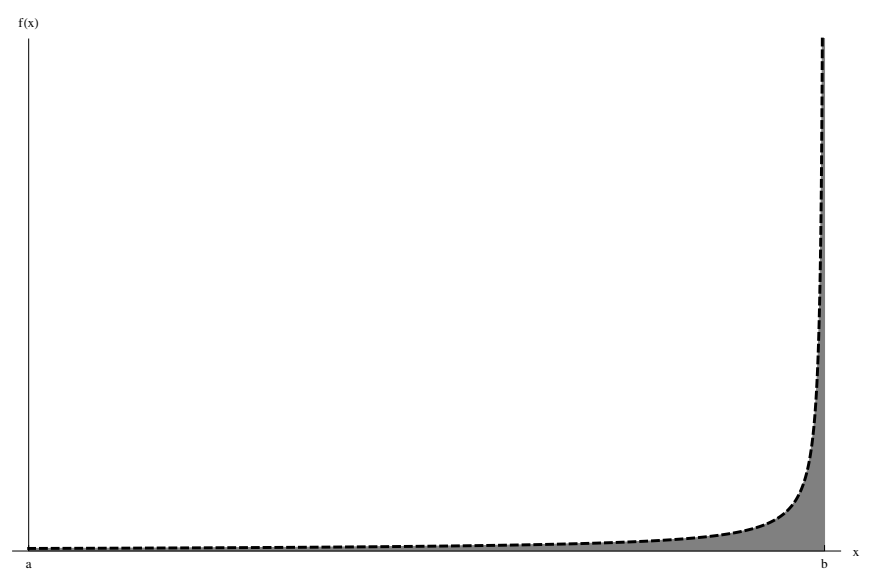

Fig. 8. Graph of the original core integrand in the formulas (1)-(3)

The transformations expressions are as follows:

$$
\begin{aligned}
& x=\phi(t)= \\
& \quad=\tanh t^{p}, \phi^{\prime}(t)=\frac{p t^{p-1}}{\cosh ^{2} t^{p}}, p=1,3,5, \ldots
\end{aligned}
$$

$$
\begin{aligned}
x= & \phi(t)= \\
& =\tanh (\phi / 2 \sinh (t)), \phi^{\prime}(t)=\frac{\phi / 2 \cosh t}{\cosh ^{2} \sinh (t)}
\end{aligned}
$$

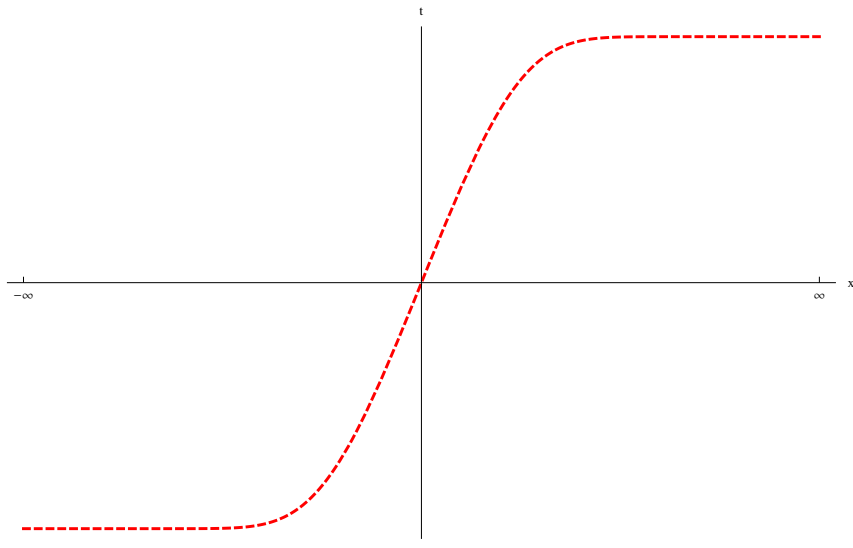

Fig. 9. Graph of the transforming expression (18)

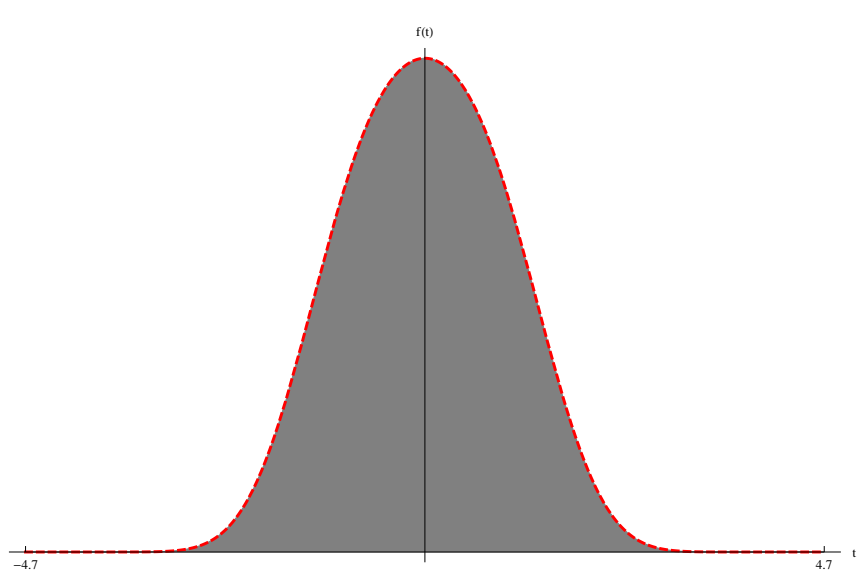

Fig. 10. Graph of the transformed core integrand (20) and the range applied in computations

$$
\begin{aligned}
x=\phi(t)=\tanh \left(\phi / 2 \sinh \left(t^{3}\right)\right), \\
\phi^{\prime}(t)=\frac{3 \pi / 2 t^{2} \phi / 2 \cosh t^{3}}{\cosh ^{2} \sinh \left(t^{2}\right)}
\end{aligned}
$$

Applying the transformation (18) to the formulas (1)-(3) according the formula (16), we obtain the following trapezoidal form

$$
S=h \sum_{i=1}^{N} f\left(\frac{b-a}{2} x_{i}+\frac{b+a}{2}\right) w_{i}
$$

where

$$
x_{i}=f\left(\tanh \left(\pi / 2 \sinh \left(t_{i}\right)\right)\right)
$$

are the nodes and

$$
w_{i}=\frac{\cosh \left(t_{i}\right)}{\cosh ^{2} \pi / 2 \sinh t_{i}} \cdot \frac{b-a}{2}
$$

are the weights of the Double Exponential Quadrature.

Additionally $t_{i}=-t_{a}+(i-1) \cdot h, i=0,1,2,3, \cdots N-$ $1, h=\frac{2 t_{a}}{N-1}$ are the new integration range and the width of one trapezoidal subinterval. The selection of the value $t_{a}$ parameter decides how near the singularity we integrate. 
There is presented the visualization of the DE transformation on Figs 8-10.

\section{B. Gauss-Jacobi Quadrature with Adopted Weight Function}

A weight function, which enables to eliminate definite integration range endpoints singularities is Jacobi weight (21) [1], [2], [3], [4].

$$
p(x)=(1-x)^{\lambda}(1+x)^{\beta}, \lambda, \beta>-1 .
$$

A quadrature formula with the weight (21) assumes the form

$$
\begin{aligned}
\int_{-1}^{1}(1-x)^{\lambda}(1+x)^{\beta} \cdot f(x) d x & \cong \\
& \cong \sum_{k=1}^{n} A_{k} \cdot f\left(x_{k}\right) .
\end{aligned}
$$

The nodes $x_{i}$ are zeros of the Jacobi polynomial $J_{n}(x ; \lambda, \beta)$.

The Jacobi Polynomial can be determined by applying Rodrigues formula

$$
\begin{aligned}
& J_{n}(x ; \lambda, \beta)= \\
& =\frac{(-1)^{n}}{2 n \cdot n !}(1-x)^{-\lambda}(1+x)^{-\beta} . \\
& \cdot \frac{d^{n}}{d x^{n}}\left[(1-x)^{\lambda+n}(1+x)^{\beta+n}\right]
\end{aligned}
$$

The weights $A_{k}$ can be computed applying the following formula

$$
\begin{aligned}
A_{k}=2^{\lambda+\beta+1} \frac{\Gamma(\lambda+n+1)}{n ! \Gamma(\lambda+\beta+n+1)} & \frac{1}{\left(1-x_{k}^{2}\right)\left[J_{n}^{(\lambda, \beta)^{\prime}} x_{k}\right]^{2}}
\end{aligned}
$$

The remainder of the Gauss-Jacobi Quadrature is expressed as

$$
\begin{aligned}
R= & \frac{2^{\lambda+\beta+2 n+1}}{\lambda+\beta+2 n+1} \\
& \frac{\Gamma(\lambda+n+1) \Gamma(\beta+n+1) \Gamma(\lambda+\beta+n+1)}{\Gamma^{2}(\lambda+\beta+2 n+1)} \\
& \cdot \frac{n !}{2 n} \cdot f^{2 n}(\xi), \xi \in\langle-1,1\rangle
\end{aligned}
$$

Now the transformation of the weight function. Substituting $\lambda=1-\alpha, \beta=0$ in (22) we obtain

$$
\int_{-1}^{1} \frac{\phi(x)}{(1-x)^{1-\alpha}} d x
$$

which coincides with the core integrand in the formulas (1)-(3).

To change the integration range from $[-1,1]$ to arbitrary chosen $\left[t_{0}, t\right]$ formula (26) must be transformed as follows

$$
\left(\frac{t-t_{0}}{2}\right)^{\nu} \cdot \int_{-1}^{1} \frac{\phi(u)}{(1-u)^{1-\alpha}} d u
$$

where

$$
\phi(u)=f\left(\left(\frac{t-t_{0}}{2}\right) u+\left(\frac{t-t_{0}}{2}\right)\right)
$$

Applying formulas (26)-(27) we can express the formula (1) as

$$
{ }^{R L} \mathbf{I}^{(\nu)}=\frac{1}{\Gamma(\nu)}\left(\frac{t-t_{0}}{2}\right)^{\nu} \int_{t_{0}}^{t} \frac{f(u)}{(t-u)^{1-\alpha}} d u .
$$

To calculate non-integer (fractional) order derivatives applying formula (3) we proceed the similar way

$$
\left(\frac{t-t_{0}}{2}\right)^{n-\nu} \int_{-1}^{1} \frac{\phi(u)}{(1-u)^{1-\alpha}} d u
$$

where

$$
\phi(u)=f\left(\left(\frac{t-t_{0}}{2}\right) u+\left(\frac{t-t_{0}}{2}\right)\right) .
$$

The formula (3) assumes the following form

$$
{ }^{C} \mathrm{D}^{(\nu)}=\frac{1}{\Gamma(n-\nu)}\left(\frac{t-t_{0}}{2}\right)^{n-\nu} \int_{t_{0}}^{t} \frac{\left(\frac{d}{d t}\right)^{n}}{(t-u)^{n-\alpha-1}} d u \text {. }
$$

The formula (28) seems to have similar form as (3). The most difficult part in context of numerical integration, however is calculated applying a method which guarantees multiple times higher accuracy, applying the Jacobi polynomials. 